\title{
Monitoring and Analysis of Drug Resistance of Yersinia in Fresh Meat
}

\author{
Liu Guihua ${ }^{1}$, Zhang Weiyu ${ }^{1}$, Huang Xin ${ }^{1}$, Gong Yunwei ${ }^{2}$ \\ ${ }^{1}$ Jilin Provincial Center for Disease Control and Prevention, Changchun, China \\ ${ }^{2}$ Changchun City Center for Disease Control and Prevention, Changchun, China
}

\section{Email address:}

liugh0523@sina.com (Liu Guihua)

\section{To cite this article:}

Liu Guihua, Zhang Weiyu, Huang Xin, Gong Yunwei. Monitoring and Analysis of Drug Resistance of Yersinia in Fresh Meat. Science Discovery. Vol. 9, No. 2, 2021, pp. 32-36. doi: 10.11648/j.sd.20210902.12

Received: March 10, 2021; Accepted: March 19, 2021; Published: March 30, 2021

\begin{abstract}
Objective To monitor the drug resistance status of yersinia isolates from fresh meat, grasp the drug resistance spectrum and trend of Yersinia isolates in Our province, and provide important reference data for guiding the rational application of antibiotics in human clinical practice and controlling the spread of drug resistant strains. Methods A total of 600 pieces of fresh pork, beef and chicken were collected from several farmers' markets and supermarkets in Changchun every month for 15 months from August 18th to October 19th. 80 strains of Yersinia strains were detected, and MIC concentrations of drug resistance were detected by microbroth dilution with 15 antibiotic sensitive plates. Results 80 strains of Yersinia strains were counted. The total drug resistance rate of Yersinia pestis was 98.8\%, and cefazolin (CFZ) had the highest drug resistance rate of 92.5.\% (74/80). Erythromycin (ERY) was followed by 58.8\% (47/80). Ampicillin (AMP) 57.5\% (46/80); Naproxil acid (NAL) and cefoxitin (CFX) were $28.8(23 / 80)$ and were somewhat resistant to other antibiotics. But all strains were sensitive to imipenem (IMP) and gentamicin (GEN). There were 28 strains with multiple drug resistance, accounting for 35.4\% (28/79). Conclusion There are multiple antibiotic resistance in Yersinia strains in this monitoring, and the multiple drug resistance is serious and the resistance spectrum is diverse. In order to ensure food safety and human health, great attention should be paid to and enhanced drug resistance monitoring of Yersinia.
\end{abstract}

Keywords: Fresh Meat Food, Yersinia, Drug Resistance Monitoring, Result Analysis

\section{鲜肉食品中耶尔森菌抗药性监测及结果分析}

\author{
刘桂华 ${ }^{1}$, 张炜煜 ${ }^{1}$, 黄釒致 ${ }^{1}$ 龚云伟 ${ }^{2}$ \\ 1吉林省疾病预防控制中心, 长春, 中国 \\ ${ }^{2}$ 长春市疾病预防控制中心, 长春, 中国
}

\section{邮箱}

liugh0523@sina.com (刘桂华)

\begin{abstract}
摘要: 目的 对来源于鲜肉食品中耶尔森菌分离株的耐药状况进行监测, 掌握我省耶尔森菌的耐药谱和耐药趋势, 为指 导抗生素在人类临床中的合理应用，控制耐药菌株的传播提供重要的参考性数据。方法 从18年 8 月到 19 年10月历经15 个月,每个月采集长春市多家农贸市场和超市销售的鲜猪肉、牛肉和鸡肉共 600 份。从中检测到的耶尔森菌菌株 80 株, 利用微量肉汤稀释法, 用含有 15 种抗生素的药敏板进行了抗药性最低抑菌浓度 (MIC) 检测。结果 对 80 株耶尔森菌抗 药性进行统计，检出耶尔森菌总耐药率为 $98.8 \%$, 其中耐药率最高的为头孢唑林（CFZ） 92.5.\%（74/80）; 其次是红 霉素 (ERY) 58.8\% (47/80); 氨苄西林 (AMP) 57.5\% (46/80); 䒺啶酸 (NAL) 和头孢西丁 (CFX) 为28.8（23/80）, 对其他抗生素有一定程度的耐药。但所有菌株对亚胺培南和庆大霉素全部敏感。多重耐药有28株，占（28/79）35.4\%。
\end{abstract}


结论 本次监测耶尔森菌存在多种抗生素的耐药, 多重耐药严重, 抗药谱多样; 应高度重视和加强耶尔森菌菌的耐药性 监测,以保证食品安全和人类健康。

关键词：鲜肉食品, 耶尔森菌, 抗药性监测, 结果分析

\section{1. 概述}

耶尔森菌属（Yersinia）属于肠杆菌科，包括鼠疫耶 氏菌、小肠结肠炎耶氏菌与假结核耶氏菌等十余个菌种, 这是一类革兰阴性小杆菌。其中鼠疫耶氏菌、小肠结肠炎 耶氏菌与假结核耶氏菌对人类的致病性已明确[1-3]。耶尔 森氏菌为动物肠道寄生菌, 广泛存在于动物体内。它是目 前国际社会广泛关注的一种食源性病原菌。该菌广泛分布 于自然界, 是能在冷藏温度下生长的少数肠道致病菌之一 [4-6]。目前在我国食品中耶尔森菌的检出率相对较低, 系 统的监测数据不多。为了解耶尔森菌菌株在食品中的污染 状况, 以及其抗药性, 掌握我省耶尔森菌的耐药谱和耐药 趋势, 为指导相应的食源性疾病的临床治疗, 本实验室受 国家食品安全评估中心委托,开展了本次监测。现将结果 报告如下。

\section{2. 材料与方法}

\section{1. 材料}

\subsection{1. 菌株来源}

80 株耶尔森菌菌株均来自于长春市多家农贸市场和 超市采样, 生猪肉 300 份 (肉馅或分割肉)、牛肉 150 份 (肉 馅或分割肉) 、整鸡 150 只, 共计 600 份样品, 通过实验室 检测获得。

\subsection{2. 培养基和试剂}

耶尔森菌选择性琼脂培养基购于英国OXOID公司; 耶 尔森菌显色培养基购于法国科玛嘉公司; CIN-I购于广州 环凯生物有限公司; 蛋白胨山梨醇胆汁肉汤购于美国 SIGMA-ALORICH公司; 尿素培养基购于北京路桥技术有 限公司; API20E 购于法国梅里埃; 小肠结肠炎耶尔森菌 标准菌株CMCC52203; 细菌基因组DNA提取试剂盒; PCR 检测所需试剂由国家食品安全评估中心提供; 冻干型细菌 定量药敏(MIC) 测试盒由上海星佰生物技术有限公司生产。 所有培养基均在有效期内。质控菌株大肠埃希菌(ATCC 25922) 购买于广州环凯生物技术有限公司。

\section{2. 方法}

\subsection{1. 耶尔森菌的检测}

各种鲜肉经处理后加入增菌液于 $4^{\circ} \mathrm{C}$ 中冷增菌 20 天; 使用普通PCR方法对冷增菌20天后的样品增菌液进行耶 尔森菌初篮; 取PCR方法检测出目的基因片段的样品增菌 液 $0.5 \mathrm{~m}$ 加入到含有 $4.5 \mathrm{~mL}$ 碱液的螺旋管中摇匀进行碱液处 理; 碱处理液

再接种于耶尔森菌选择性分离培养基培养; 分离平板 上可疑菌落经分解尿素的菌株阳性培养物, 使用 API $20 \mathrm{E}$ 生化试剂条鉴定进行系统生化鉴定。所有耶尔森菌属的菌 株, 均需保留进行药敏试验。

\subsection{2. 药敏试验}

按照美国国家临床实验室标准委员会, 纸片法抗菌药 物敏感试验标准(NCCLS \CISI 2007), 采用肉汤稀释法 MIC板 [7-9]。将质控菌株和备检菌株分别接种于哥伦比亚 血琼脂平板, $37{ }^{\circ} \mathrm{C} 24 \mathrm{~h}$ 纯培养。挑取 $3 \sim 5$ 个纯新菌 落, 均匀悬浮于 $10 \mathrm{~mL}$ 生理盐水制成细菌稀释管中, 再将 该菌液调整于 0.5 麦氏比浊管使菌液的细菌含量达到 $1 \times 10^{8} \mathrm{CFU} / \mathrm{ml}$; 首先在生物安全柜内使用微量移液器吸取 已比浊好浓度的菌液 $10 \mu \mathrm{l}$ 加入到 $12 \mathrm{ml}$ 肉汤管中混匀, 并缓慢倾倒入 $\mathrm{V}$ 型无菌槽内, 再使用八道微量移液器依 次加入到 96 孔药敏板中(每孔 $100 \mu \mathrm{l})$ 用塑料盖盖好, 接种 过程完毕, 最终接种浓度为 $1 \times 10^{5} \sim 2 \times 10^{5} \mathrm{CFU} / \mathrm{ml}$ 左右。 阳性对照孔加入 $100 \mu \mathrm{l}$ 菌悬液即可, 整个过程注意无菌 操作。将接种完毕后的药敏试剂盒放入 $36 \pm 1{ }^{\circ} \mathrm{C}$ 温箱中 16 18 h。在祄有黑底板的光线下, 用肉眼观察。阳性对 照孔混浊表明细菌生长良好, 凡细菌生长的小孔中, 呈弥 散状浑浊或小孔底部有圆形或网状沉淀。细菌生长的孔内 所含最低抗菌药物浓度即为最低抑菌浓度(MIC)。

\section{3. 结果}

\section{1. 本次实验使用的药敏板的质控情况}

耶尔森菌药敏试验板室内质控报告[10], 质控菌株试 验板的结果在质控范围内。（见表1）

表1 质控菌株大肠埃希菌 ATCC25922 的 MIC 值 允许范围和 MIC 解释标准.

\begin{tabular}{|c|c|c|c|c|}
\hline \multirow{2}{*}{ 抗生素名称 } & \multirow{2}{*}{$\begin{array}{l}\text { MIC 范围大肠埃希菌 } \\
\text { ATCC25922 }\end{array}$} & \multicolumn{3}{|c|}{ MIC 解释标准 $(\mu \mathrm{g} / \mathrm{ml})$} \\
\hline & & 敏感 S & 中介 IR & 耐药 \\
\hline 氨苄西林 $\mathrm{AMP}$ & $2 \sim 8$ & $\leq 8$ & 16 & $\geq 32$ \\
\hline 氨芐西林/舒巴坦AMS & $2 / 1 \sim 8 / 4$ & $\leq 8 / 4$ & $16 / 8$ & $\geq 32 / 16$ \\
\hline 头孢唑啉 CFZ & $1 \sim 4$ & $\leq 2$ & 4 & $\geq 8$ \\
\hline 头狍他啶 CAZ & $0.06 \sim 0.50$ & $\leq 4$ & 8 & $\geq 16$ \\
\hline 四环素 TET & 0. $5 \sim 2$ & $\leq 4$ & 8 & $\geq 16$ \\
\hline 红霉素 ERY & & $\leq 0 . \quad 5$ & $1 \sim 4$ & $\geq 8$ \\
\hline
\end{tabular}




\begin{tabular}{|c|c|c|c|c|}
\hline \multirow{2}{*}{ 抗生素名称 } & \multirow{2}{*}{$\begin{array}{l}\text { MIC 范围大肠埃希菌 } \\
\text { ATCC25922 }\end{array}$} & \multicolumn{3}{|c|}{ MIC 解释标准 $(\mu \mathrm{g} / \mathrm{ml})$} \\
\hline & & 敏感 $\mathbf{S}$ & 中介 I R & 耐药 \\
\hline 环丙沙星 CIP & $0 . \quad 004 \sim 0.015$ & $\leq 0.06$ & $0.12 \sim 0 . \quad 50$ & $\geq 1$ \\
\hline 萘啶酸 NAL & $1 \sim 4$ & $\leq 16$ & & $\geq 32$ \\
\hline 头狍西丁 CFX & $2 \sim 8$ & $\leq 8$ & 16 & $\geq 32$ \\
\hline 头狍噻肜 CTX & $0.03 \sim 0.12$ & $\leq 1$ & 2 & $\geq 4$ \\
\hline 庆大霉素 GEN & $0.25 \sim 1$ & $\leq 4$ & 8 & $\geq 16$ \\
\hline 复方磺胺 SXT & $\leq 0.5 / 9.5$ & $\leq 2 / 38$ & & $\geq 4 / 76$ \\
\hline 氯霉素 CHL & $2 \sim 8$ & $\leq 8$ & 16 & $\geq 32$ \\
\hline 亚胺培南 IMP & $0 . \quad 06 \sim 0.25$ & $\leq 1$ & 2 & $\geq 4$ \\
\hline 阿奇霉素AZM & & $\leq 8$ & 16 & $\geq 32$ \\
\hline
\end{tabular}

注: 没有中介浓度是由于敏感和耐药两个浓度为相邻的倍比浓度。

\subsection{0株耶尔森菌的肉汤稀释法 MIC 药敏状况}

对 80 株耶尔森菌抗药性进行统计, 检出耶尔森菌总耐 药率为 $98.8 \%$, 其中耐药率最高的为头狍唑林 (CFZ) $92.5 . \%$ (74/80)；其次是红霉素 (ERY)58.8\%、(47/80);氨芐西
林 (AMP) $57.5 \%(46 / 80)$; 菜啶酸 (NAL) 和头孢西丁 (CFX) 为28.8 (23/80), 对其他抗生素有一定程度的耐药; 但所 有菌株对亚胺培南和庆大霉素敏感。（见表2）

表2 80 株食源性耶尔森菌肉汤稀释法 MIC 药敏结果。

\begin{tabular}{|c|c|c|c|c|c|}
\hline 抗生素 & 敏感数 (株) & 中介数 (株) & 耐药数 (株) & 耐药率 (\%) & MIC 值 $(\mu \mathrm{g} / \mathrm{ml})$ \\
\hline 氨苄西林 AMP & 9 & 25 & 46 & 57.5 & $4 \sim>64$ \\
\hline 头孢他啶CAZ & 78 & 0 & 2 & 2.5 & $\leq 1 \sim>32$ \\
\hline 氨苄西林/舒巴坦AMS & 48 & 27 & 5 & 6.3 & $\leq 2 / 1 \sim 64 / 32$ \\
\hline 亚胺培南 IMP & 80 & 0 & 0 & 0 & $\leq 0.25$ \\
\hline 四环素 TET & 68 & 4 & 8 & 10.0 & $\leq 1 \sim>32$ \\
\hline 䒬啶酸 NAL & 57 & 0 & 23 & 28.8 & $\leq 2 \sim>64$ \\
\hline 红霉素 ERY & 1 & 32 & 47 & 58.8 & $\leq 0.5 \sim>16$ \\
\hline 头狍西丁 $\mathrm{CFX}$ & 40 & 17 & 23 & 28.8 & $\leq 2 \sim>64$ \\
\hline 氯霉素 CHL & 77 & 1 & 2 & 2.5 & $\leq 2 \sim>64$ \\
\hline 头孢噻朊 $\mathrm{CTX}$ & 75 & 2 & 3 & 3.8 & $\leq 0.25 \sim>8$ \\
\hline 头孢唑啉 CFZ & 3 & 3 & 74 & 92.5 & $\leq 0.5 \sim>16$ \\
\hline 庆大霉素 GEN & 80 & 0 & 0 & 0 & $\leq 1 \sim>32$ \\
\hline 复方磺胺 SXT & 78 & 0 & 2 & 2.5 & $\leq 0.25 / 0.475 \sim>8 / 152$ \\
\hline 阿奇霉素AZM & 78 & 0 & 2 & 2.5 & $\leq 4 \sim>64$ \\
\hline 环丙沙星 CIP & 2 & 24 & 4 & 5.0 & $\leq 0.03 \sim 2$ \\
\hline
\end{tabular}

\subsection{0 株耶尔森菌的耐药菌株的耐药谱}

除 1 株无抗药性外,其他分成 18 种耐药谱。其中对 1 种 抗生素耐药24株, 分成3种抗药谱; 对2种抗生素耐药 27 株, 分成 2 种抗药谱; 对 3 种抗生素耐药17株, 分成3种抗 药谱; 对4种抗生素耐药2株, 分成2种抗药谱; 对5种抗生
素耐药4株, 分成4种抗药谱; 对6种抗生素耐药1株, 分成 1 种抗药谱; 对7种抗生素耐药 1 株, 分成 1 种抗药谱; 对 8 种抗生素耐药3株, 分成2 种抗药谱。抗药谱分布最多的是 CFZ 19株; 其次是AMP-CFZ-16株，多重耐药株从 $3 \sim 8$ 种抗生素不等。（见表3）

表3 80 株耶尔森菌的耐药菌株的耐药谱。

\begin{tabular}{|c|c|c|c|}
\hline 抗生素种类 & 抗药谱 & 株数 & 合计 \\
\hline \multirow[t]{2}{*}{0} & & 1 & 1 \\
\hline & CFZ-、 & 19 & \\
\hline \multirow[t]{2}{*}{1} & NAL-、 & 2 & 24 \\
\hline & AMP- & 3 & \\
\hline \multirow{3}{*}{2} & AMP-CFZ-、 & 16 & 27 \\
\hline & NAL-CFZ- & 11 & 21 \\
\hline & AMP-CFX-CFZ-、 & 14 & \\
\hline \multirow[t]{2}{*}{3} & AMP-TET-CFZ-、 & 2 & 17 \\
\hline & AMP-CAZ-CFZ- & 1 & \\
\hline \multirow{3}{*}{4} & AMP-NAL-CFZ-CIP、 & 1 & 2 \\
\hline & AMP-NAL-CFX-CFZ- & 1 & \\
\hline & AMP-AMS-CFX-CTX-CFZ- & 1 & \\
\hline \multirow{3}{*}{5} & AMP-AMS-TET-NAL-CFZ--、 & 1 & 4 \\
\hline & AMP-TET-NAL-CFX-CFZ- & 1 & 4 \\
\hline & AMP-NAL-CFX-CFZ-AZM- & 1 & \\
\hline 6 & AMP--AMS--NAL-CFX--CFZ--CIP & 1 & 1 \\
\hline
\end{tabular}




\begin{tabular}{llll}
\hline 抗生素种类 & 抗药谱 & 株数 & 合计 \\
\hline 7 & AMP--TET-NAL-CFX-CHL-CTX-CFZ & 1 & 1 \\
8 & AMP-AMS-TET-NAL-CFX-CFZ-SXT-CIP- & 2 & 3 \\
\hline
\end{tabular}

\section{4. 耶尔森菌耐药性较高的五种抗生素的 $\mathrm{MIC}(\mu \mathrm{g} / \mathrm{mL})$ 值分布}

林MIC值 $16 \mu \mathrm{g} / \mathrm{mL} \sim 64 \mu \mathrm{g} / \mathrm{mL}$ 占 $83.8 \%$ (67/80）; 芸啶酸 氨䒾西林 $\mathrm{MIC}$ 值 $\leq 2 \mu \mathrm{g} / \mathrm{mL}$ 占 $65.0 \%(52 / 80)$; 头孢西丁 $\mathrm{MIC}$

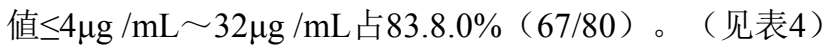

头狍唑林MIC值 $>16 \mu \mathrm{g} / \mathrm{mL}$ 占 $63.8 \%$ (51/80) ; 红霉 素MIC值 $4 \mu \mathrm{g} / \mathrm{mL} \sim 16 \mu \mathrm{g} / \mathrm{mL}$ 占 $92.5 \%$ (74/80); 氨芐西

表4 耶尔森菌耐药性较高的抗生素的 $\mathrm{MIC}(\mu \mathrm{g} / \mathrm{ml})$ 值分布.

\begin{tabular}{|c|c|c|c|c|c|c|c|c|c|}
\hline 头孢唑林 & & 红霉素 & & 氨芐西林 & & 䒬啶酸 & & 头孢西 & \\
\hline MIC值 & 样品（份） & MIC值 & 样品（份） & MIC值 & 样品（份） & MIC值 & 样品 (份) & MIC值 & 样品 (份) \\
\hline$\leq 0.5$ & 2 & $\leq 0.5$ & 1 & $\leq 2$ & 0 & $\leq 2$ & 52 & $\leq 2$ & 9 \\
\hline 1 & 0 & 1 & 1 & 4 & 2 & 4 & 3 & 4 & 12 \\
\hline 2 & 1 & 2 & 0 & 8 & 7 & 8 & 1 & 8 & 19 \\
\hline 4 & 3 & 4 & 32 & 16 & 25 & 16 & 1 & 16 & 17 \\
\hline 8 & 16 & 8 & 25 & 32 & 20 & 32 & 0 & 32 & 19 \\
\hline 16 & 7 & 16 & 17 & 64 & 22 & 64 & 5 & 64 & 4 \\
\hline$>16$ & 51 & $>16$ & 4 & $>64$ & 4 & $>64$ & 18 & $>64$ & 0 \\
\hline 合计 & 80 & & 80 & & 80 & & 80 & & 80 \\
\hline
\end{tabular}

\section{4. 讨论}

\section{1. 食品中耶尔森菌耐药状况及其监测意义}

耐药（R）：表示按常规给药计划通常可达到的药物 浓度不能抑制其生长的菌株或证实其 MIC 值落在可能存 在微生物特殊耐药机制（如产 $\beta$-内酰胺酶）的范围内, 临 床治疗研究显示该药物对菌株的临床疗效并不可靠。本次 监测来源于食源性疾病的 80 株耶尔森菌耐药情况比较严 重 [11-13], 对80株耶尔森菌抗药性进行统计, 检出耶 尔森菌总耐药率为 $98.8 \%$, 其中耐药率最高的为头孢唑林 (CFZ) 92.5.\%（74/80）；其次是红霉素 (ER Y)58.8\%、 (47/80); 氨苄西林（AMP)57.5\%(46/80); 䒺啶酸（NAL） 和头孢西丁 (CFX) 为28.8（23/80），对其他抗生素有一 定程度的耐药; 但全部菌株对亚胺培南和庆大霉素敏感。 $\mathrm{MIC}$ 值 $\leq 2 \sim>64(\mu \mathrm{g} / \mathrm{mL})$ 不等，结果表明来源于食源性疾 病的耶尔森菌的耐药性是较强的, 在临床治疗中要根据抗 生素的敏感性合理的选择使用药物, 才能达到治疗效果。

\section{2. 建立耐药谱的意义}

本次监测28株多重耐药, 其中对3种抗生素耐药17株, 分成3种抗药谱; 对4种抗生素耐药2株, 分成2种抗药谱; 对5种抗生素耐药4株, 分成4种抗药谱; 对6种抗生素耐药 1 株, 分成1种抗药谱; 对7种抗生素耐药1株, 分成1种抗 药谱; 对8种抗生素耐药3株, 分成2种抗药谱。抗药谱分 布最多的是CFZ 19株; 其次是AMP-CFZ-16株，多重耐 药株从 $3 \sim 8$ 种抗生素不等。抗药谱一致表明抗药株可能 为同一来源, 为食源性疾病的溯源有一定的意义。多重耐 药严重这与我国目前的现实情况相符, 大多数病人去医院 就医都是因为就医前使用抗生素不见好转, 因为感染的菌 对多种抗生素耐药, 腹泻症状较重而就医。目前多重耐药
给食源性疾病的临床治疗工作造成较大的困难, 值得有关 部门高度重视 [14-15]。

\section{3. 耐药菌株的MIC值分布}

MIC 是指在琼脂和肉汤稀释药物敏感性试验中抑制 细菌可见生长的某种抗菌药物的最低浓度。同一细菌对不 同药物的敏感性用MIC 值衡量, 其值越小, 说明越敏感, 其抑菌作用越强。本次监测菌株耐药性较高的五种抗生素 的 $\mathrm{MIC}(\mu \mathrm{g} / \mathrm{mL})$ 值分布: 头孢唑林MIC值 $>16 \mu \mathrm{g} / \mathrm{mL}$ 占 $63.8 \%(51 / 80)$; 红霉素MIC值 $4 \mu \mathrm{g} / \mathrm{mL} \sim 16 \mu \mathrm{g} / \mathrm{mL}$ 占 $92.5 \%$

(74/80); 氨䒾西林MIC值 $16 \mu \mathrm{g} / \mathrm{mL} \sim 64 \mu \mathrm{g} / \mathrm{mL}$ 占 $83.8 \%$ (67/80); 䒺啶酸氨芳西林 $\mathrm{MIC}$ 值 $\leq 2 \mu \mathrm{g} / \mathrm{mL}$ 占 $65.0 \%$ (52/80) ; 头孢西丁 $\mathrm{MIC}$ 值 $\leq 4 \mu \mathrm{g} / \mathrm{mL} \sim 32 \mu \mathrm{g} / \mathrm{mL}$ 占 $83.8 \%$ (76/80)。MIC越大值大, 分布越集中说明该菌耐药上升 的趋势越明显。

\section{5. 结论}

通过各种食源性致病菌的耐药性监测, 建立统一的抗 生素耐药监测数据库, 表明我省肉类食品中耶尔森菌的抗 药性与其他食品中常见的致病菌抗药性较强, 头孢唑林 (CFZ); 红霉素 (ER Y); 氨苠西林 (AMP); 萫啶酸 (NAL) 和头孢西丁 (CFX)临床治疗效果不佳, 但亚胺培南 (IMP) 和庆大霉素 (GEN) 会有很好的治疗效果; 试验结果表明 其耐药性不仅较高, 并且存在一定的多重耐药株; MIC越 大较大, 并且分布集中, 说明该菌耐药上升的趋势越明显。 本次监测为建立统一的抗生素耐药监测数据库, 通过各种 食源性致病菌的耐药性监测, 指导临床医生合理有效使用 抗生素, 可提高细菌耐药性的检测能力和水平, 掌握我省 主要食源性致病菌耐药趋势, 对食源性疾病的预警、预防 和治疗具有非常重要的指导意义 [16]。 


\section{基金项目}

吉林省卫生技术创新项目（2016J034）。

\section{参考文献}

[1] 刘桂华, 黄金金, 王艳秋, 张炜显等.长春市肉类食品中耶尔 森菌污染状况的检测及其结果分析 [J]. Asia-Pacific Journal of Food Science and Technology亚太食品科学与技术期刊, 2020; 2(1): $1-5$

[2] 陈邬锦, 王鹏. 中国小肠结肠炎耶尔森菌流行现状及其研究 进展 [J].中国人兽共患病学报,2015,31(04):380-384。

[3] 郑浩轩,姜泊.小肠结肠炎耶尔森菌研究概况[J].中国微生态 学杂志.2006.18(5):416-419。

[4] 黄英, 景怀琦.耶尔森菌侵袭性研究进展[J].中国人畜共患病 学报,2009,25(7):669-672。

[5] 王金鍂.中国小肠结肠炎耶尔森菌分子流行病学研究[D].中国 疾病预防控制中心.2009。

[6] 王晓英.生奶中小肠结肠炎耶尔森菌和嗜水气单胞菌的复 合和半巢穴 PCR 检测方法 [J]. 国外医学卫生学分册, 2002,2(2):127-128。

[7] 李顺姬, 王树东, 曹铁红, 等. 41株致泻大肠埃希菌的药 敏试验检测及结果分析 [J]. 中国卫生工程学, 2016,15(2):127-129.

[8] 肖丹, 刘桂华, 曲莉, 等. 肉汤稀释法 M I C 药敏试剂盒 对沙门菌检测结果分析 [J]. 中国卫生工程学, 2014,13(4):314-315,319.

[9] 李月婷, 龚云伟, 刘桂华. 吉林省 20014 年食源性金黄 色葡萄球菌的耐药性分析 [J]. 中国卫生检验杂志, 2015,25(21):3772-3774.
[10] 李顺姬, 刘桂华. 食品中单核细胞增生李斯特菌的抗药性 检测及结果分析 [J]. 中国卫生检验杂志, 2015,25(20), 3586-3587.

[11] 李月婷, 龚云伟, 刘桂华. 食源性沙门菌和致泻性大肠杆 菌的耐药性分析 [J] . 中国卫生检验杂 志,2016,26(22):3335-3336.

[12] 刘桂华, 黄金金, 张炜显, 等. 食品中金黄色葡萄球菌和单 核细胞增生李斯特菌抗药性检测及结果分析 $[\mathrm{J}]$. 中国卫生 工程学, 17(4):497-500.

[13] Liu Guihua, Huang Xin, Zhang Weiyu, 等.Detection and Analysis of Antimicrobial Resistance of Salmonella in Faeces of Foodborne Disease[J]. PatientsScience Journal of Public Health 2020; 8(3): 77-82

[14] 章志超,龙慧, 吴金鍂,刘德.食品中小肠结肠炎耶尔森氏菌分离 鉴定方法研究进展 [J]. 食品安全质量检测学 报,2020,11(02):455-461。

[15] 王爱华,赵德东,韦杰.散装生肉中小肠结肠炎耶尔森氏菌的 污染状况分析 [J].大家健康(学术版),2014,8(11):26-27。

[16] 张蕴哲,杨倩,马晓燕,杨澜,张伟.RF-LAMP技术检测鸡肉中 小肠结肠炎耶尔森氏菌 [J]. 食品研究与 开发,2018,39(04):133-138。

\section{作者简介}

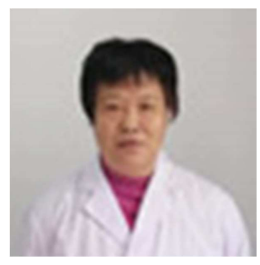

刘桂华（1962-）女, 主任医师, 主要 从事微生物检验及方法研究工作。 